\title{
Five dimensional cosmological models in Lyra geometry with time dependent displacement field
}

\author{
G. Mohanty $\cdot$ K.L. Mahanta $\cdot$ B.K. Bishi
}

Published online: 26 July 2008

(C) Springer Science+Business Media B.V. 2008

Erratum to: Astrophys Space Sci (2007) 310: 273-276

DOI 10.1007/s10509-007-9513-9

(1) Equation (11) in page 274 i.e.

$$
\frac{B^{\prime}}{B}\left[\frac{3 A^{\prime}}{A}+\frac{B^{\prime \prime}}{B^{\prime}}\right]=0
$$

be replaced by

$$
\frac{3 A^{\prime} B^{\prime}}{A B}+\frac{B^{\prime \prime}}{B}=0
$$

(2) Equation (16) in page 274 i.e.

$$
\frac{A^{\prime}}{A}\left[\frac{2 A^{\prime}}{A}+\frac{A^{\prime \prime}}{A}\right]=0
$$

be replaced by

$$
2\left(\frac{A^{\prime}}{A}\right)^{2}+\frac{A^{\prime \prime}}{A}=0 .
$$

(3) In the Subcase 3.1.3, "This subcase leads to Subcase 3.1.1" be replaced by "This case is not acceptable as the second term becomes indeterminate".

(4) Case 3.3 in page 275, "In this case we get same result as obtained in Sect. 3.1.1" be replaced by "This case is not acceptable as the second term becomes indeterminate".

(5) Equation (37) in page 275 i.e.

$\frac{B^{\prime}}{B}\left[3 n \frac{B^{\prime}}{B}+\frac{B^{\prime \prime}}{B^{\prime}}\right]=0$

be replaced by

$$
3 n\left(\frac{B^{\prime}}{B}\right)^{2}+\frac{B^{\prime \prime}}{B}=0 .
$$

(6) The Subcase 4.2.3 in page 276, "This subcase leads to the Subcase 4.2.1" be replaced by "This case is not acceptable as the second term becomes indeterminate".

The online version of the original article can be found under doi:10.1007/s10509-007-9513-9.

G. Mohanty $(\bowtie) \cdot$ K.L. Mahanta $\cdot$ B.K. Bishi

P.G. Department of Mathematics, Sambalpur University, Jyoti Vihar 768019, Orissa, India

e-mail: ganeshwarm@yahoo.com 